Revista de Matemática: Teoría y Aplicaciones 2007 14(1) : 21-44

CIMPA - UCR - CCSS ISSN: 1409-2433

\title{
ESTUDIO DE OPINIÓN SOBRE EL T.L.C. CON EE.UU.
}

\author{
Jorge POLtronieri*
}

Recibido/Received: 4 Dic 2006 - Aceptado/Accepted: 31 Jan 2007

\section{Resumen}

En este trabajo se exponen los resultados de una encuesta de opinión sobre el Tratado de Libre Comercio con EE.UU. La técnica del Análisis en Componentes Principales nos ayudó a revelar la opinión subyacente en la comunidad costarricense. Los resultados muestran una gran preocupación en la población sobre las implicaciones del tratado, en la sociedad.

Palabras clave: Estructuras de opinión, encuesta de opinión pública, análisis en componentes principales, libre comercio.

\begin{abstract}
We expose the results of an opinion survey on the Free Trade Agreement with the United States of America. The technique of Principal Component Analysis helped us to reveal the subjacent opinion in the Costarican comunity. The results show us a great population's preocupation about the implications of the trade, in the society.
\end{abstract}

Keywords: Opinion structures, public opinion survey, principal component analysis, free trade.

Mathematics Subject Classification: 62H25, 91B14.

\section{Introducción}

Mucho se ha hablado en los medios de comunicación de las bondades del Tratado de Libre Comercio (T.L.C.) con Estados Unidos (EE.UU.). Sin embargo, ningún estudio se había llevado a cabo sobre este tema, indagando de manera exhaustiva sobre los pro y los contra de este tratado, al menos en los que hasta ahora se han mostrado en la prensa del país.

${ }^{*}$ CIMPA \& Escuela de Matemática, Universidad de Costa Rica, 2060 San José, Costa Rica. E-Mail: jpoltro@costarricense.cr 
El estudio formó parte de un estudio anual que se realiza en el país desde hace veinte años, en lo que atañe al problema del estudio de la Opinión Pública. En el 2006 nos dimos a la tarea planificar una batería de preguntas, lo más completa posible, que abordara los pro y los contra del tratado. Para esto, se contó con las colaboración de valiosos académicos en el campo de la política, sociología, geografía, historia, economía, derecho y de la opinión pública. El resultado del esfuerzo concluyó con veintiséis preguntas, que abordan los temas más importantes que se relacionan con los consecuencias que nos traerá el tratado a corto y mediano plazo.

Los resultados obtenidos en este estudio, son de una importancia extraordinaria, toda vez que nos arrojan luz sobre los sentimientos y suspicacias que despiertan el tratado de libre comercio, en la población costarricense. En ningún otro estudio, se observa de manera tan clara y precisa, los sentimientos que los costarricenses tienen en torno a este tratado.

Sabemos que la metodología usada es la causante de tan importante hallazgo, pues nuestra una faceta que no es fácil de observar a simple vista en los resultados, pero que está latente en las respuestas que la población ofreció en este estudio. Pero también debe darse crédito a lo atinado de las preguntas y la claridad de las mismas, lo que permitió llegar a tan importantes conclusiones.

\subsection{Procedimiento del muestreo}

Marco muestral: Dado que el presente trabajo trata de estudiar la estructura de la opinión pública de Costa Rica, la muestra debía ser elaborada a nivel nacional. Se fijó el tamaño de la muestra en 1000 individuos y se determinó la composición de la misma.

Del último censo de población (2000) se extrajeron los siguientes datos:

- Población total por provincia.

- Población total por cantón de cada provincia.

- Población total por distrito de cada cantón.

Como siguiente paso, se calcularon las relaciones porcentuales:

- De la población total de cada provincia con respecto a la población nacional.

- De la población total de cada cantón con respecto a la población total de la provincia.

A partir de los datos anteriores, fueron seleccionados los cantones y distritos que presentaron mayor concentración poblacional y que fuesen representativos de su provincia en cuanto a la distribución por sexo y por edad de la población.

El factor concentración poblacional es relevante para el estudio, pues los entrevistados deben tener acceso a los medios de comunicación colectiva. Son los criterios anteriores los que permitieron la escogencia de los segmentos censales.

La encuesta propiamente dicha se lleva a cabo con entrevistadores previamente entrenados para tal fin, que visitan, respetando cuotas, las personas en su domicilio, para 
entrevistarlas individualmente. La entrevista tiene una duración de unos treinta y cinco minutos. Los datos socio-demográficos y socio-culturales son tomados en cuenta en el cuestionario.

El encuestador, marca directamente sobre el cuestionario la respuesta del entrevistado. Después de la eliminación de los cuestionarios dudosos y de llevar a cabo una verificación general de las encuestas, se procede a su análisis.

En lo que se refiere a la muestra, se respeta el efectivo de individuos en diversas categorías (de acuerdo a los datos del último censo nacional u otra fuente confiable), tales como:

- La estratificación general por región: cuotas por provincias.

- Dos cuotas específicas:

Sexo.

La categoría socio-profesional del entrevistado:

* Agricultores.

* Patrón de empresa y comercio.

* Cuadros superiores y profesionales liberales.

* Maestros y profesores.

* Empleados.

* Estudiantes.

* Pensionados.

* Amas de casa.

* Sin profesión.

Hemos dado aquí una posible agrupación de las diversas categorías socio-profesionales. Es posible considerar otras alternativas.

\section{La Base de Datos}

Las preguntas del cuestionario se agrupan en tres rúbricas:

\subsection{Primera rúbrica: La señalización}

La señalización se caracteriza fundamentalmente por obtener indicadores socio-demográficos o socio-culturales de los entrevistados. La región, el sexo, la edad, la profesión, corresponden a estas características, así como el salario, el nivel de estudios, la tendencia política, el estado civil, la religión, el empleo y algunas otras. La profesión del entrevistado es una de las variables fundamentales para la señalización, pues en el estudio de las estructuras de la opinión pública no se trata de estudiar el comportamiento económico de grupos sociales, sino sus mentalidades. 


\subsection{Segunda rúbrica: Los medios de comunicación}

Se encuentran aquí de una manera general preguntas relativas a la televisión, radio y periódicos. En los tratamientos estadísticos estas variables figuran, como en la señalización, en calidad de variables ilustrativas.

\subsection{Tercera rúbrica: Preguntas de opinión sobre el TLC}

Las preguntas se presentan, al igual que los temas de conflicto, bajo la forma de proposiciones. Las respuestas de estos temas son analizadas a la luz de los resultados sobre los temas de conflicto. Un total de 26 preguntas se consideraron.

\section{Bases teóricas y metodológicas}

En esta parte se describe la metodología de construcción de lo que puede llamarse un barómetro multidimensional de opinión. Para esto debe tenerse en consideración las siguientes interrogantes: ¿Cuáles son las ideas fundamentales, las hipótesis, para construir este barómetro? ¿Cómo el recurso del lenguaje de los sistemas y la geometría permite ligarlas a las restricciones que impone la cuantificación? ¿Qué lógica sostiene el procedimiento utilizado para la construcción de cuestionarios? ¿Cómo interpretar los indicadores producidos? Abordaremos en primera instancia una teoría general de conflictos. Algunos encontrarán aquí la discusión eterna entre el cielo (los mitos, los héroes, los dioses) y la tierra (las costumbres, las opiniones) en que se ha debatido la humanidad a través de su historia.

El conflicto, como lugar privilegiado de observación de las opiniones, nos indica el procedimiento para resaltar las estructuras de la diferenciación entre individuos. Las dimensiones que permiten desdoblar los conflictos y analizar las opiniones, son producidas recurriendo a la vez al modelo factorial (Charles Spearman) y el análisis en componentes principales (Karl Pearson).

\subsection{Teoría de conflictos y esquema de la economía de roles: el conflicto como modo de negociación y regulación social}

Para intentar explicar los mecanismos de la opinión, se mencionan aquí los primeros elementos de una teoría general de conflictos. Según esta teoría, los conflictos de nuestras sociedades, que se discuten a través de los medios de comunicación colectiva, tienen una triple función:

- Legitimar las grandes decisiones imponiendo el debate entre instituciones.

- Armonizar los cambios institucionales y la evolución de las mentalidades.

- Permitir a los individuos comunicarse y adquirir un cierto conocimiento.

Así por ejemplo, la aceptación del público de nuevas tecnologías al nivel de un país dependerá, al menos en parte, de la eficacia de regulación social asegurada por los conflictos 
que ellas provocan. El papel del Estado está en definir las reglas que permitan el debate entre los portavoces de las instituciones que toman parte en el conflicto.

\subsubsection{Dos sistemas de interacción: las instituciones y el público}

Se observa entre las fuerzas sociales que se oponen ante un conflicto, todo un conjunto de instituciones: estado, parlamento, empresas, sindicatos, asociaciones ...todas suponen intereses compartidos u objetivos comunes con el público. Los individuos intervienen a otro nivel, pues son las interacciones con el medio que entran en juego y no la sicología individual. El público a venido a ser un factor decisivo en todas las sociedades modernas: juega un rol de árbitro omnipresente en las grandes decisiones que son tomadas a nivel de las principales instituciones. Estas a su vez tienen una influencia sobre las mentalidades, las actitudes y los comportamientos de los individuos. Estos dos sistemas bien diferentes - las instituciones y el público - evolucionan juntos, por lo que hacer proyecciones de uno u otro exige que se conozcan más sus mecanismos de interacción.

\subsubsection{En sus interacciones, las instituciones se transforman al ritmo de los conflictos}

Si vamos más allá de una simple negociación, el conflicto — que es llevado de alguna manera a la plaza pública - indica que ciertas instituciones (en sus acciones, sus relaciones interinstitucionales o en su existencia misma) están amenazadas. En el origen del conflicto se encuentra un proyecto, un problema, en breve un hecho que para algunos es doloroso o escandaloso. Esto provoca discusiones vivas que oponen a lo que llamamos actores sociales y hacen evidente el conflicto. Los actores son los portavoces de las instituciones que toman parte. Los discursos, los argumentos que se desarrollan traducen los roles que ellos tienen: estamos en la escena de un teatro. Esta escena, no es otra que la de los medios de comunicación colectiva.

\subsubsection{Para el público el debate entre actores es ante todo un espectáculo}

En general el hombre de la calle, el individuo social, no es parte ejecutante de los hechos: no participa en las decisiones políticas. No es el individuo quien decide la política financiera de las instituciones, no está encargado del financiamiento de la seguridad social ..., o está lejos del lugar donde son tomadas las decisiones para que pueda tener una influencia determinante. La mayoría de las veces sus decisiones no trascienden más allá de cuestionar su confort, su vida, pues parte del conocimiento de los protagonistas; tan sólo lo asiste un sentimiento de duda y de contradicción. ¿Cómo tener un criterio comedido de un conjunto de afirmaciones opuestas, a menudo enfáticas y perentorias?

En fin, no se trata de escoger entre lo falso o lo verdadero, sino aceptar o rechazar algo que se revela bueno o malo, o bien escoger entre el bien y el mal. Si bien todo está ahí para excitar el interés del hombre común, nada se hace para incitarlo a la búsqueda de la verdad, abstrayéndole de sus sentimientos, sus reflejos y sus temores. Y sin embargo, ¿quién no ha dado su opinión e igualmente argumentado en pro o en contra cerca del problema de la educación? Claramente los conflictos, interesando a la opinión pública, son una ocasión para comunicarse unos con otros. El conflicto, que provoca el debate entre actores, es 
ante todo para el individuo espectador un tema de expresión. Pero entonces, ¿qué hay de común entre el espectáculo de la escena, es decir, el debate entre actores sociales y la conversaciones animadas que son la extensión entre el público? ¿Cómo los actores se ajustan al público y recíprocamente?

\subsubsection{El conflicto es un medio para medir la correlación de fuerzas}

A nivel de los actores, ¿qué interesa que el hombre común esté o no impregnado del problema que se debate? Lo esencial es que un arbitraje opera entre las fuerzas ...y he ahí el rol de la "vox pópuli" a través de la prensa y las encuestas. Todo sucede como si existiera un tribunal público donde la argumentación producida será analizada en su justo valor. No hay jueces en el público, pero esta ilusión se lleva a la sociedad entera. La lucha provocada por el problema y esta ilusión van a permitir a los actores clarificar las premisas, afinar la doctrina y concretar el razonamiento. Los conflictos aportan más que algunos indicadores sobre el derrumbe del edificio institucional (relegados a un segundo plano en el momento de una elección donde se oponen permanentemente las fuerzas políticas); son un modo de administración de la correlación de fuerzas, que permite respetar los grandes principios que han conducido al sufragio universal.

\subsubsection{El conflicto es también un medio de comunicación que permite la adquisición de un cierto conocimiento}

Para algunos, la búsqueda del reconocimiento de otros (la afirmación de su estatus) es suficiente para explicar las discusiones animadas que tienen lugar en el público ...y los resortes de orden sicológico revelan la economía interna del ser. Pero estas conversaciones tienen un papel social, pues van a permitir a las mentalidades adaptarse a las transformaciones institucionales.

Para llegar al conocimiento, algunos argumentan que hay que tener fe. La fe es la fuerza que hace (en el círculo social que lo solicita) que la balanza se torne en favor de unos y en detrimento de otros. Ella se expresa en la toma de posición, que puede ser considerada como una primera fase efectiva en la respuesta al conflicto. Es la información que recibe de los medios, la que hace posible este primer ajuste. Sin punto de referencia, el conflicto no puede por si sólo operar como objeto de diferenciación entre individuos. La discusión va a permitir consolidar la fe. ¿No es la conversación el lugar propicio donde, bajo la presión de otros, se encuentra la facultad de ordenar las ideas? Así, la oscuridad puede acceder a una cierta claridad. Los argumentos producidos por los actores, van a ser reforzados o debilitados, para tener un hilo conductor que dará al individuo social la apariencia de tener la razón. El argumento a posteriori es sacado de esta segunda fase, más evaluativa de la respuesta a la situación que impone el conflicto. Este argumento, salido de un examen crítico, indica que hay interiorización de una lógica y por tanto permite el acceso a un cierto conocimiento. Así el debate entre actores se prolonga entre el público. El nuevo espectáculo que se desarrolla es en apariencia idéntico al que se desarrolla en los medios de comunicación. Como consecuencia de los ajustes que se operan en el momento de la comunicación, los papeles no son distribuidos al azar. Ellos son escogidos en función 
del lugar que ocupa o que quiere ocupar el individuo en el universo social. Es apropiándose de un rol que se dice a otro dónde está situado, qué lo diferencia. Es así como se efectúa el desarrollo de las opiniones.

\subsubsection{Esquema de economía de roles}

En las sociedades democráticas modernas, los grandes conflictos que aparecen en los medios de comunicación colectiva juegan un papel importante: son los motores de un cambio social que se puede realizar así, sin sacudidas violentas. Y sin embargo, el desorden que evidentemente provocan puede ser creador de un nuevo equilibrio más satisfactorio.

En efecto, al imponer el debate entre instituciones, los conflictos permiten explicar las grandes alternativas nacionales, armonizar los cambios institucionales, los cambios tecnológicos y la evolución de las mentalidades y le brindan incluso la posibilidad a los individuos de comunicarse entre ellos para tener acceso a un cierto conocimiento.

Pero, ¿cuáles son los mecanismos que, gracias a estos conflictos, hacen evolucionar las cosas suavemente y respetando los grandes principios de la democracia? Como veremos, estos mecanismos dependen de lo que se llama una economía de roles.

En una primera aproximación, las opiniones pueden considerarse como reflejo de un equilibrio análogo al existente en el mercado, pero será necesario adaptar el esquema habitual al que hace referencia la economía liberal, cuando se refiere a los productos correspondientes las necesidades. Recordemos que este esquema reposa sobre dos clases de actores: productores y consumidores. El equilibrio, si se logra, es considerado como el resultado de dos clases de interacciones: con la primera - la competencia - los productores se enfrentan entre ellos; con la segunda - el juego de la oferta y la demanda- se enfrentan productores y consumidores. En el caso más simple, se supone que los consumidores actúan aisladamente y su acción se deduce, como la de los productores, de una lógica: la de la ganancia.

Con los conflictos, se pasa del mercado al espectáculo. Igualmente hay dos clases de protagonistas implicados: los actores sociales - que son los voceros de los organismos e instituciones que toman parte en el conflicto- y el público. La competencia entre actores se evidencia en el debate que se plantea; se producen argumentos reflejando los intereses alrededor de los cuales se desarrolla el conflicto. Pero, teniendo lugar en parte en la prensa y los otros medios de comunicación colectiva, el debate se convierte para el público en un espectáculo. Se produce entonces el juego de la oferta y la demanda, puesto que el rol jugado por el actor, en interacción con los otros participantes, debe tomar en cuenta la atención del público.

Aquí, el esquema simple de la economía de mercado se complica; en realidad el individuo no actúa aisladamente. Una tercera interacción interviene, visible en las discusiones que prolongan el debate entre actores hacia el público. El conflicto se convierte para el individuo espectador en lugar de proyección y de identificación. La toma de posición es una ocasión de afirmar el yo, de decir al otro dónde se sitúa uno con respecto a él; esta apropiación inconsciente de uno de los roles jugados por los actores se deriva, por lo tanto, no solamente de la oferta, sino también de esta interacción con los otros que se llama la diferenciación. Esta toma de posición en el debate a favor o en contra de las 
privatizaciones, a favor o en contra del parlamento centroamericano, etc.) provoca, en el juego interior, un ordenamiento de los argumentos producidos por los actores; hay en este estadio, interiorización de una lógica y por lo tanto acceso a un cierto conocimiento que basta para dar a nuestro individuo social más que la apariencia de la razón.

He aquí cómo se efectuaría el deslizamiento de las opiniones: coincidiría con la apropiación permanente por parte de los individuos de los roles que se le ofrecen en el espectáculo que crean los conflictos, renovados sin cesar.

Lo considerado anteriormente nos permite hablar de una economía de roles. Es el triple juego de las interacciones siguientes:

- Entre actores sociales: el debate.

- Entre actores y el público: el juego de la oferta y de la demanda de roles.

- Entre individuos sociales: la diferenciación que lleva a las sociedades democráticas modernas hacia nuevos equilibrios, bajo el impulso de los conflictos, donde el debate entre instituciones y las opiniones quedan en correspondencia con sus diversidades.

El esquema de economía de roles es una respuesta global a la pregunta: ¿Cómo se efectúa la comunicación, en países como el nuestro, cuando se produce un conflicto?

En el confinamiento de una primera fase del examen de un macro-sistema es característica la confrontación entre instituciones y el público - cuando esta relación es puesta en escena de manera espectacular por los medios de comunicación-. Esta confrontación indica cuáles son los dos sub-sistemas (los actores y el público) que es necesario considerar en primera instancia para comprender el fenómeno, y cuáles son las fuerzas, las interacciones, que aseguran que este sistema evolucione su cohesión: estamos aquí ante un proceso dinámico de aprendizaje.

El recurso del lenguaje permite a todas estas fuerzas manifestarse y así, autorizando los ajustes, asegurar la convergencia hacia nuevos equilibrios. Los puntos de vista y los argumentos producidos frente al público por los actores no son más que los resultados de una negociación, que se desarrolla en forma permanente entre las organizaciones antagonistas. A través de sus portavoces cada uno de los actores busca mejorar o defender su posición. Los individuos proceden de una manera equivalente: cuestionar un actor, contrarrestar su argumento, es decir su rol, es también hacer una escogencia estratégica en la relación que se desarrolla con otros individuos. Esto lleva a resaltar o por el contrario a borrar una diferencia: se trata también de confrontar una posición.

\subsection{Análisis de la diferenciación: modelo factorial y análisis en componentes principales}

Consideramos los conflictos como lugar privilegiado de observación de la opinión. Con el esquema de la economía de roles disponemos de elementos (conceptos y relaciones entre conceptos) suficientes para considerar la construcción de un barómetro multidimensional.

Las dimensiones que permiten desdoblar los conflictos y analizar las opiniones son producidas recurriendo a la vez al modelo factorial (Charles Spearman) y al análisis en componentes principales (Karl Pearson). 
Siguiendo un enfoque clásico en psicometría, los modelos arriba citados proveen de herramientas metodológicas para la obtención de indicadores que se puedan considerar característicos de la diferenciación. Estos indicadores son retenidos para analizar los conflictos, las opiniones y sus evoluciones.

\subsubsection{Un nuevo objeto: la interacción}

Cuando se hace referencia al individuo a través de un conflicto, se hace explícitamente referencia a:

- Por un lado: el conflicto.

- Por otro lado: el individuo social, es decir el individuo tomado en su relación con otros.

La teoría de sistemas enseña que a estos dos elementos hay que agregar un tercero:

- La interacción.

$$
\text { conflicto } \longrightarrow \text { interacción } \longleftarrow \text { individuo social }
$$

Para describir el conflicto, tomado aisladamente, el sociólogo dispone de un enfoque que puede calificarse de denotativo, el cual consiste en señalar las situaciones alrededor de las cuales se desarrolla la controversia entre las partes. Igualmente para describir el individuo social, tomado aisladamente, el sico-sociólogo puede apoyarse en la noción de estatus, la cual permite caracterizar los diferentes roles jugados por los individuos en su vida social. Pero aquí estos dos elementos no son tratados separadamente. Para describir su interacción, debe tomarse en cuenta dos nociones (una relacionada con la otra):

- La connotación: traduce el verdadero significado que da el individuo social al conflictoestímulo.

- La actitud de la diferenciación: es la predisposición que provoca el conflicto en el individuo.

Esta predisposición consiste en utilizar la ocasión para expresar su diferencia, es decir, su estatus. Tomando en cuenta la interacción, se puede hacer corresponder por dualidad conflictos e individuos sociales. Si el conflicto engendra las actitudes individuales, éstas recíprocamente dan un significado al conflicto.

Para prever comportamientos individuales, se podrá partir de la connotación: ¿cuál es el significado social el conflicto? Para encontrar el significado de un conflicto, se podrá partir de las actitudes de diferenciación: ¿qué indica el hombre común cuando utiliza el conflicto para comunicarse? 


\subsection{El recurso del Modelo Factorial}

Suponer que son siempre los mismos mecanismos que entran en juego cuando el individuo toma una decisión, conduce a considerar un modelo de la forma:

$$
y=f(d, s)
$$

donde, $y$ representa la posición del individuo frente al conflicto, $d$ y $s$ son los conjuntos de parámetros que permiten traducir, en su interacción, actitudes de diferenciación y connotaciones respectivamente. Dentro del modelo, los mecanismos que conducen a la toma de posición, que suponemos idénticos cualquiera que sea el conflicto y el individuo considerado, se representan por el funcional $f$ :

$$
\begin{array}{ccc}
\text { conflicto } & f \\
\text { denotación } & \longrightarrow \text { interacción } \longleftarrow \quad \begin{array}{c}
\text { individuo social } \\
\text { estatus }
\end{array} \\
y=f(d, s) . &
\end{array}
$$

Así, los conflictos pueden ser asociados a los pares $(y, s)$ y los individuos sociales a los pares $(y, d)$.

No conocemos a priori el funcional $f$. No se conoce tampoco los conjuntos de parámetros $d$ y $s$. Una aproximación va permitir utilizar el modelo lineal, en el cual quedan por determinarse los parámetros.

Si nos colocamos en un vecindario del individuo $\left(y_{0}, d_{0}\right)$, el modelo (1) se escribe (utilizando un desarrollo limitado de primer orden):

$$
y-y_{0}=f_{d}^{\prime}\left(d_{0}, s\right)\left(d-d_{0}\right)+\text { residuo. }
$$

Seleccionamos $n$ individuos $\left(y_{i}, d_{i}\right)$ considerados como parte del vecindario de su centro de gravedad $(\bar{y}, \bar{d})$ y $p$ estímulos $\left(y^{j}, s^{j}\right)$. Se trabaja bajo la hipótesis que, cualquiera que sea el estímulo, la derivada de $f$ con respecto a $d$ es constante alrededor del vecindario. Se obtiene entonces de (2) el modelo lineal:

$$
x_{i}=S c_{i}+\mathrm{e}_{i}=\sum_{l=1}^{k} c_{i}^{l} s_{l}+\mathrm{e}_{i}, \quad i=1, \ldots, n .
$$

En este modelo:

- $x_{i}=y_{i}-\bar{y}$ es el vector de $\mathrm{R}^{p}$ cuyas coordenadas son las respuestas centradas $x_{i}^{j}=y_{i}^{j}-\bar{y}^{j}$ del $i$-ésimo individuo a los $p$ conflictos-estímulo.

- $S$ es la matriz $p \times k$ asociada a la matriz Jacobiana $f_{d}^{\prime}$. Sus columnas $s_{l}$ engendran en $\mathrm{R}^{p}$ los $k$ ejes de diferenciación buscados.

- $c_{i}=d_{i}-\bar{d}$, representa el $i$-ésimo individuo cuando se señala en el sistema de ejes de diferenciación. Sus coordenadas son los valores centrados $c_{i}^{l}=d_{i}^{l}-\bar{d}$. 
- $\mathrm{e}_{i}$ es el vector de errores $\mathrm{e}_{i}^{j}$ en la aproximación de $x_{i}$.

De manera análoga, las ecuaciones (3) se escriben:

$$
x^{j}=C s^{j}+\mathrm{e}^{j}=\sum_{l=1}^{k} s_{l}^{j} c^{l}+\mathrm{e}^{j}, \quad j=1, \ldots, p,
$$

donde:

- $x^{j}$ es la variable centrada asociada al $j$-ésimo conflicto, de coordenadas $x_{i}^{j}=y_{i}^{j}-\bar{y}^{j}$.

- $C$ es la matriz $n \times k$ de coeficientes $c_{i}^{l}$, cuyas columnas $c^{l}$ engendran en $\mathrm{R}^{n} \operatorname{los} k$ ejes de las connotaciones buscadas.

- $s^{j}$ es el vector de coordenadas $s_{l}^{j}$. Módulo una constante de proporcionalidad, estos valores serán considerados como coordenadas del $j$-ésimo conflicto en el sistema de $k$ ejes de las connotaciones.

- e $\mathrm{e}^{j}$ es el vector de errores $e_{i}^{j}$ de la aproximación de $x^{j}$.

Los sistemas de ecuaciones (3) y (4) corresponden a dos escrituras equivalentes del modelo factorial de Charles Spearman.

\subsection{La búsqueda de parámetros del modelo factorial: El Análisis en Componentes Principales}

¿Cómo encontrar los parámetros $c^{l}$ y $s_{l}$ del modelo lineal? Más precisamente, si se hace referencia al sistema de ecuaciones (3), ¿cómo calcular la matriz $S$ y las coordenadas $c_{i}$ de los individuos en el sistema de ejes de diferenciación? La explotación de ciertas propiedades que deben verificar los residuos van a permitir llevar esta búsqueda a la solución de un problema de optimización. Se tiene entonces que el análisis factorial permite obtener la solución. La escogencia de la técnica de análisis depende del criterio usado.

\subsubsection{Independencia de los residuos: según C. Spearman y L. Thurstone}

La independencia entre residuos es una exigencia clásica en estadística, cuando se juzga la adecuación del modelo. Lo que se deja de lado, es lo que se conoce en teoría de señales como ruido de fondo. Desde un punto de vista geométrico, esta exigencia impone en la ecuación (4) la ortogonalidad (correlación nula), entre las variables $c^{l}$ y las variables $x^{j}$ por un lado, y los residuos e $\mathrm{e}^{j}$ por otro lado. Cuando se considera la noción de correlación parcial, éstas condiciones se expresan así: las correlaciones parciales, condicionadas a las variables $c^{l}$, entre las variables $x^{j}$ y $x^{j^{\prime}}$ son iguales a cero, es decir:

$$
\operatorname{cor}\left(x^{j}, x^{j^{\prime}} / c^{1}, c^{2}, \ldots, c^{k}\right)=0, \quad j \neq j^{\prime}
$$

El modelo de Spearman, o modelo en factores comunes y específicos, se tiene a través de las ecuaciones (4) completadas con las ecuaciones (5). Este modelo refleja una situación ideal, que sugiere el problema de optimación siguiente: buscar, en el espacio $\mathrm{R}^{n}$ de $n$ 
dimensiones, el subespacio $W$ de dimensión $k$, tal que las proyecciones de las variables $x^{j}$ sobre el ortogonal $W^{\perp}$ de $W$ (de los residuos e ${ }^{j}$ ) sean lo más ortogonales posible. Toda la clase de $W$ define entonces un conjunto optimal de componentes factoriales $c^{l}$. Varios criterios han sido propuestos para hacer mínimas las correlaciones entre residuos, por ejemplo el criterio del determinante máximo. Este consiste en maximizar, el determinante de la matriz de correlaciones entre residuos. A los criterios corresponden un conjunto de procedimientos iterativos de extracción de componentes factoriales.

\subsubsection{Minimilidad de los residuos: según K. Pearson y H. Hotelling}

Para encontrar los parámetros $c^{l}$ y $s^{l}$, K. Pearson propuso en 1901, ajustar la nube de puntos $x_{i}$ de $\mathrm{R}^{p}$. Las variables centradas reducidas y la distancia euclídea clásica se escogen para medir a proximidad en $\mathrm{R}^{p}$, los ejes optimales definen el subespacio buscado (son los vectores propios de la matriz de correlación). Aquí se utiliza el análisis en componentes principales. Hotelling en 1933 descubre que el análisis en componentes principales da solución al siguiente problema: sabiendo que $\operatorname{cor}^{2}(x, c)$ designa el coeficiente de correlación entre las variables $x$ y $c$, encontrar as $k$ variables ortogonales (no correlacionadas) $c^{l}$ que maximizan sucesivamente la cantidad:

$$
J=\sum_{j} \operatorname{cor}^{2}\left(x^{j}, c\right)
$$

La simplicidad de los cálculos del análisis en componentes principales tiene la ventaja de proveer soluciones $s_{l}$ y $c^{l}$ que se corresponden por dualidad. El argumento de dualidad precedente justifica la escogencia del Análisis en Componentes Principales (A.C.P.) como modelo factorial de extracción de parámetros del modelo mecanismos de la diferenciación).

\section{La construcción del cuestionario}

Las $k$ dimensiones $c^{l}$ y $s_{l}$ obtenidas por el Análisis en Componentes Principales son por definición las características de la diferenciación. No deben éstas depender de los individuos interrogados ni de las variables escogidas. El sondeo debe efectuarse según cuotas y los entrevistados se consideran como el resultado de un muestreo al azar en la población estadística (la forman los costarricenses de más de 18 años que viven en ciudad). Los resultados del análisis factorial dependen sólo de la población de referencia (no dependen de los $p$ estímulos-conflicto: estos se muestrean al azar, dentro de una población estadística representativa del conjunto de conflictos). El problema que se presenta es que no podemos sacar al azar preguntas de un universo que no sabemos precisar. Para visualizar esta situación recordemos las paradojas que se discutían antes de la axiomatización de la teoría de probabilidades por Kolmogorov: sacar al azar en un universo de conflictos provisto de una medida de probabilidad es equivalente a darse un procedimiento fijo de producción de conflictos, que siendo aleatorio, respeta ciertas reglas. Con base en lo anterior el procedimiento que utilizamos opera en tres etapas:

- Selección de los temas a tratar. 
- Selección por un grupo de expertos los temas a considerar.

- Construcción, alrededor de los temas, de las preguntas referéndum que no sean ambiguas.

\section{Análisis en Componentes Principales}

Se consideraron las preguntas sobre el TLC (26 en total), para el análisis en componentes principales. Procedimos de manera que las no respuestas de los temas se reemplazaron por las medias de los mismos, con el fin de que no influenciaran las correlaciones. Un análisis en componentes principales se efectúa sobre las respuestas de los temas de conflicto del cuestionario. Recordemos que este análisis conduce a extraer los vectores propios de la matriz de correlación. La tabla de datos centrada individuos $\times$ variables que se ha analizado comprende 1000 líneas (los individuos entrevistados) y 26 columnas (26 temas de sobre el TLC). A esta tabla se le asocian dos nubes de puntos:

- la primera de 1000 individuos, está situada en un espacio de 26 dimensiones

- la segunda de 26 variables, se sitúa en un espacio de 1000 dimensiones.

Los vectores propios de la matriz de correlación generan los ejes principales de inercia de la nube de individuos. Estos ejes jerarquizados, definen un nuevo sistema de referencia ortogonal en el espacio de 26 dimensiones. A los ejes principales les corresponde, por dualidad, las componentes principales (combinaciones lineales de las variables iniciales) que son ortogonales (no correlacionadas); definiendo éstas un nuevo sistema de coordenadas en el espacio de los 1000 individuos. Las coordenadas de las variables en este nuevo sistema, son sus correlaciones con las componentes principales. Los valores propios de la matriz de correlación son iguales a las varianzas de las componentes principales. Ellas miden la dispersión de los individuos a lo largo de los ejes. La suma de los valores propios, que es igual a 26, mide la dispersión total de la nube de individuos alrededor de sus centros de gravedad (varianza total). Entre más grande es la dispersión de los individuos a lo largo de un eje principal, mayor será el porcentaje de varianza total explicada. El subespacio de dimensión $k$ que mejor ajusta la nube de individuos en el espacio de 26 dimensiones, es el que engendra los $k$ primeros ejes principales; la dispersión de la nube en este subespacio se mide por la suma de los $k$ primeros valores propios. Cuanto más el subespacio está próximo a la nube de puntos, tanto mayor será el porcentaje de varianza explicada. Habiendo escogido la dimensión del espacio principal, nos interesamos en las descripciones de las dos primeras dimensiones factoriales, luego en la tercera y cuarta, etc. Sobre los planos definidos por las componentes principales (círculo de correlaciones), se examinan las posiciones ocupadas por los temas de conflicto. ¿Cuáles son las connotaciones? Sobre los planos generados por los ejes principales (planos principales), se examinan las posiciones ocupadas por los grupos sociales: la manera en que se diferencian. 


\subsection{Selección de las dimensiones principales}

Con el histograma de los diez primeros valores propios, se da la inercia explicada por cada dimensión (porcentajes) y por los sub-espacios generados por los primeros ejes principales (porcentaje acumulado). ¿Cuántas dimensiones factoriales se deben retener? La selección de las dimensiones se puede hacer examinando el histograma:

- con la regla del codo (igualdad de los últimos valores propios), se seleccionan 2 dimensiones

- con la regla del punto de inflexión (Catell) consideraríamos 2 dimensiones.

La regla del codo consiste en llegar hasta la componente en que la variación con respecto a las restantes es débil. La regla de Catell, que se basa en determinar los puntos de inflexión en los histogramas de los valores propios, llega a un resultado similar. Pruebas $F$ de Fisher se pueden realizar para evaluar la significancia de las variables de la señalización y las primeras componentes principales de valor propio superior a 1. La componente es más estable, entre mayor sea su valor propio, y de más fácil interpretación si aparece ligada a las variables de la señalización.

\begin{tabular}{r|cccl}
\hline \hline & $\begin{array}{c}\text { Valor } \\
\text { propio }\end{array}$ & & \% acum. & \\
\hline 1 & 5.448 & 20.95 & 20.95 & \\
2 & 1.962 & 7.54 & 28.50 & \\
3 & 1.685 & 6.48 & 34.98 & \\
4 & 1.411 & 5.42 & 40.40 & \\
5 & 1.122 & 4.31 & 44.72 & $\mathbf{0}$ \\
6 & 1.056 & 4.06 & 48.78 & $\mathbf{0}$ \\
7 & .979 & 3.76 & 52.54 & $\mathbf{0}$ \\
8 & .953 & 3.66 & 56.21 & $\mathbf{0}$ \\
9 & .912 & 3.50 & 59.72 & $\mathbf{0}$ \\
10 & .863 & 3.31 & 63.04 & $\mathbf{0}$ \\
\hline \hline
\end{tabular}

Tabla 1: Histograma de los 10 primeros valores propios.

\subsection{Comentarios sobre las dimensiones factoriales}

Si el cuestionario ha sido bien construido, respetando los procedimientos de tiraje al azar descrito, las dimensiones factoriales extraídas son por construcción las dimensiones de la diferenciación. Se explotan las fotografías que proveen los conflictos (círculo de correlación) y las opiniones (planos principales). Para interpretar las componentes factoriales debemos observar con más detalle los conflictos y los grupos sociales que se toman en cuenta, a fin de dar un significado concreto a las mismas. Para llevar a cabo el análisis no hemos establecido diferencias entre los conflictos, ni entre los individuos sociales. Lo esencial es que sean representativos de sus universos respectivos. Las dos primeras dimensiones 
factoriales proveen simultáneamente un mapa el cielo (círculo de correlación), en el cual los conflictos son las estrellas, y un mapa de la tierra (plano factorial) donde se encuentran los grupos sociales. Los ejes de connotación que permiten describir el cielo (componentes principales) y los ejes de diferenciación que describen la tierra (ejes principales) están en correspondencia por dualidad. Hay que contemplar simultáneamente los dos mapas para interpretar las dimensiones extraídas: los cuatro cuadrantes numerados 1, 2, 3 y 4 en los dos gráficos se corresponden. Asociándolos ligamos los grupos sociales a la opiniones sobre los conflictos que los caracterizan. Recordemos que los ejes principales son por definición y construcción los ejes de diferenciación máxima. Ellos se revelan eficaces para examinar rápidamente (economizándose el análisis de las tablas cruzadas), las relaciones existentes entre las variables de la señalización y los temas de conflictos. En el círculo de correlación del primer plano, es una verdadera fuente de información. Los temas de la periferia próximos entre sí, tienen para el público, grosso modo, el mismo significado. Sobre el plano principal, que se corresponde por dualidad al círculo de correlación, se pueden admirar las posiciones relativas ocupadas por las profesiones, grupos de edad, nivel educativo y práctica religiosa. El objetivo primordial de este método es, interpretar las componentes principales, afín de poder describir las actitudes de la población costarricense, en cuanto a la opinión pública. En la medida en que hay cierta coherencia en la expresión de las opiniones, se está tentado en investigar si hay en la expresión de las opiniones, algo más profundo y estable que llamamos actitudes. Esta noción que es teórica y técnica, resulta de los trabajos de los sicólogos sociales y se caracterizan por cuatro elementos:

1) Se trata de una variable inferida que se construye; es el factor subyacente que revela el análisis factorial y que da cuenta de un conjunto de opiniones. La actitud no es directamente observable.

2) Dado que es una disposición más o menos durable, la actitud caracteriza la persona o el grupo y no solamente sus acciones.

3) Las actitudes están generalmente polarizadas, cargadas de afectividad sobre un sujeto dado, pues ellas están en relación con las creencias y los valores. Siempre hay una actitud a favor y una actitud en contra.

4) En fin, las actitudes son adquiridas y son susceptibles a influencias externas y desde este punto de vista, la socialización no es más que la formación en el individuo de actitudes convenientes, en relación con los valores y las normas sociales de un grupo.

Esta definición permite pasar de la descripción cualitativa de una mentalidad a una verdadera medida, gracias a las técnicas de sondeo de opinión y las escalas de actitudes.

\section{El TLC con los EE.UU.}

Una observación se revela pertinente al analizar cualquier cuestión que involucra la Opinión Pública y en particular, sobre el TLC. Al preguntar ¿Se debe aprobar el TLC?, la pregunta que por sí sola no tiene validez, no quiere decir nada, pues pueden haber muchos 
motivos por los cuales una persona responda si o no. Por ejemplo, si a una persona no le gusta el TLC, pero tiene miedo que si no se firma el tratado, se pierde el comercio con EE.UU., estaría tentada decir SÍ. Por el contrario, si un individuo rechaza el TLC y está convencida que el TLC no le conviene por razones de tipo ideológico, como pueden ser razones relacionadas con la soberanía, diría que NO. Así que dos personas distintas en se manifiestan en contra del TLC, opinarían distinto sobre la aprobación del tratado por condicionamientos reales. Esto demuestra que debe realizarse toda una batería equilibrada de ítemes, donde se aborden aspectos positivos y negativos del tema tratado, con el objeto de obviar este problema. Resulta claro que deben tomarse en cuenta aspectos ideológicos, donde lo extranjero, 1 a soberanía, las costumbres, la solidaridad, la identidad como país, etcétera entren en juego. Con base en estos criterios y dado el impacto que sobre el futuro de Costa Rica tiene la aprobación del TLC, se decidió realizar una batería de 24 ítemes, más dos temas de conflicto que se preguntó en los temas de conflicto de la Encuesta Nacional. El estudio de opinión pública, sitúa al T.L.C. en un escenario de lucha ideológica sobre el futuro del país. Con excepción del COMBO, una lucha estructurada de esta envergadura no se había suscitado en nuestro país desde hace muchos años. Esta lucha opone dos conceptos de estado, dos conceptos de país. Es una lucha entre el centro y la derecha, entre el PAC y el PLN-ML, entre la soberanía y sus opositores.

Los resultados muestran un rechazo a las negociación que se dio del T.L.C., ya que promueve el intervencionismo extranjero en asuntos que son resorte de nuestra soberanía nacional. La población resiente la forma truculenta, fraudulenta, escondida y en secreto, en que se desarrolló la negociación del T.L.C., el pago de sobresueldos a los negociadores ticos por parte de los organizaciones de EE.UU. y que los EE.UU. paguen la propaganda a favor del T.L.C. que se hace en el país. La población no cree que el T.L.C. sea la única alternativa para el desarrollo de nuestro país, se critica que los medios de comunicación estén a favor del T.L.C., en una manera tan abierta, ignorando toda manifestación en contra. Tampoco le gusta que el T.L.C. esté por encima de la Constitución de la República. No cree que el T.L.C. sea la maravilla de tratado que se nos ha hecho creer, le ve problemas que afectan los intereses de sectores importantes de nuestro sistema productivo y que traerá consecuencia negativas sobre amplios sectores de la población, como el aumento de las medicinas, la privatización del ICE, INS y sobre todo del Seguro Social, con consecuencias imprevisibles sobre la salud de población. Es la centroamericanización de Costa Rica. También se debe reconocer que la encuesta nuestra una gran sabiduría del pueblo costarricense, al olfatear, intuir con "malicia indígena", que con este T.L.C. nos están vistiendo una mona con seda, nos están maquillando un engaño. A pesar de la propaganda a favor del T.L.C., una campaña donde se escucha en los medios sobre las ventajas, bondades y bellezas del T.L.C., donde incluso el Presidente de la República en su recuente viaje a Europa, pidió el apoyo al Papa para que mandara poner orden en casa, el pueblo costarricense responde de una manera vehemente y contundente, con un NO a este T.L.C., un rechazo categórico a este mundo maravilloso, casi idílico que nos va a traer este tratado. A la población todo esto le pareció y le continua pareciendo sospechoso. No es por azar que la población cree que no es correcto que con el T.L.C., se brinden derechos permanentes sobre las aguas que utilizan actualmente empresas extranjera, no le guste que con el T.L.C. algunos recursos naturales como las playas que 
actualmente son de los costarricenses, pasen a manos extranjeras, no le guste que con el T.L.C. las personas que "Queman" discos pasan a ser criminales, no le guste que con el T.L.C. las grandes empresas eliminarán a las pequeñas, no le guste que al llegar nuevas grandes empresas extranjeras con el T.L.C., el espíritu de solidaridad irá desapareciendo y tampoco le agrade la idea que el T.L.C. es un tratado que beneficia más a EE.UU. que a Costa Rica, es decir mayoritariamente opina que Debe negociarse otro T.L.C. con EE.UU. y en todo caso debe Debe hacerse un referéndum para decidir el futuro del T.L.C. con EE.UU. En resumen, podemos decir que el pueblo costarricense es un pueblo sabio, que se las sabe de todas, todas, no lo están engañando, sabe muy bien qué va a pasar con el T.L.C.; que se está vendiendo nuestra soberanía, va a destruir todo lo alcanzado por el pueblo costarricense en los últimos 60 años, lo van a tirar por el caño del desagüe, que si bien traerá algunos beneficios, este T.L.C. nos traerá, como ya se dijo anteriormente, la privatización del I.C.E., el I.N.S., de la C.C.S.S., encarecerá las medicinas, no va a proteger el medio ambiente, provocará la banca rota de los agricultores, entregará nuestra agua y nuestras playas al mejor postor extranjero. La ciudadanía desea un tratado que respete la identidad nacional, que pugna por la defensa de nuestra soberanía, donde se defiende el ser costarricense, donde nos podamos aferrar a los ideales (para algunos caducos) de equidad, solidaridad y de justicia social. Esto es lo que en realidad desea el pueblo costarricense, y no este tratado donde no se respeta la soberanía nacional y donde los negociadores y los que hoy lo promueven, no tuvieron el menor reparo en salvaguardar la dignidad de un país, no tuvieron el menor reparo en salvaguardar nuestra identidad nacional, no tuvieron el menor reparo en salvaguardar nuestras riquezas, no tuvieron el menor reparo en salvaguardar nuestros valores, tradiciones y creencias, no tuvieron el menor reparo en defender nuestra agricultura, no tuvieron el menor reparo en defender nuestro medio ambiente, no tuvieron el menor reparo en respetar la palabra empeñada que no se iban privatizar las tele-comunicaciones ni los seguros, no tuvieron el menor reparo en favorecer a los EE.UU. antes que a Costa Rica. Dicho de otro modo, este T.L.C. logra lo que no pudo el filibustero William Walker. No olvidemos que la lucha por nuestra soberanía nos costó la muerte del $10 \%$ de la población costarricense en 1856, causada por la actitud criminal y terrorista de los filibusteros. Al analizar los resultados podemos ver cómo se desplaza el nivel educativo, la edad y el ingreso. Resulta curioso que la propaganda del T.L.C. no haya permeado a los jóvenes, como si lo hizo con los grupos de mayor edad. También se observa que las personas con mayor nivel educativo rechazan mayoritariamente el T.L.C., a pesar de las promesas de mayor oportunidad para las personas con mejor preparación, hechas por los defensores del T.L.C. Esta posición no la comparten otros grupos. Tal es el caso de los grupos de más altos ingresos que se favorecen al tratado, evidenciando con esto que para ellos creen que les va a traer beneficios. Otros resultados curiosos que aparecen en este análisis tiene que ver con la práctica religiosa, ya que las personas comprometidas con la religión, como los evangélicos, protestantes y católicos practicantes y las personas sin religión, están contra el tratado. En este último grupo la motivación es ideológica. No sucede así, con los católicos no practicantes donde la posición a favor es notoria. También lo favorece la categoría Otra religión. Algo parecido sucede con el estado civil donde los solteros se oponen al tratado, en tanto que los casados, viudos y divorciados lo favorecen. Cuando analizamos las variables zona urbano-rural y provincia, la zona rural rechaza el 
tratado. En cuanto a la provincia, queda manifiesto el apoyo de Heredia y Cartago al tratado; San José un poco en contra, en tanto que Alajuela y Limón están abiertamente contra el tratado, donde el rechazo tiene un tinte ideológico. Lo mismo que Puntarenas y Guanacaste, aunque es claro que esta actitud de Puntarenas y Guanacaste, debe ser distinta (sobre todo en Guanacaste), al estar expuestas por un tiempo prolongado al turismo, donde en principio se han ido cambiando tradiciones, costumbres, valores y creencias. Producto de esto, el rechazo no es ideológico, sino una actitud cauta hacia el o lo extranjero, aunque si se aceptan ciertas actitudes que riñen con la soberanía. La política juega un papel muy importante al analizar el tratado, pues desde el punto de vista ideológico el PAC, Ningún partido y los que se denominan de Izquierda se oponen al trío PLN, PUSC y ML y a la Derecha. Cuando se toma en cuenta la profesión, se observa que los maestros, profesores, estudiantes, intelectuales, guarda público, profesionales del sector público, las amas de casa solas, las amas de casa cuyo esposo es un empleado o pensionado y la empleada doméstica son grupos que se oponen al tratado. No así, los obreros calificados, los choferes, las profesiones liberales, las amas de casa cuyo compañero es empresario o de profesión liberal, el técnico o profesional medio, el guarda privado, los empleados del sector privado, los pensionados, el pequeño y mediano comerciante, el pequeño y mediano agricultor, los empresarios, los profesionales del sector privado y la administración pública y empresa se manifiestan a favor del tratado. Se observa entre las preguntas realizadas en los años 2004, 2005 y 2006, que el T.L.C. va perdiendo terreno entre los costarricenses, por lo que una discusión más profunda se impone ante las dudas sobre las bondades que tiene este tratado.

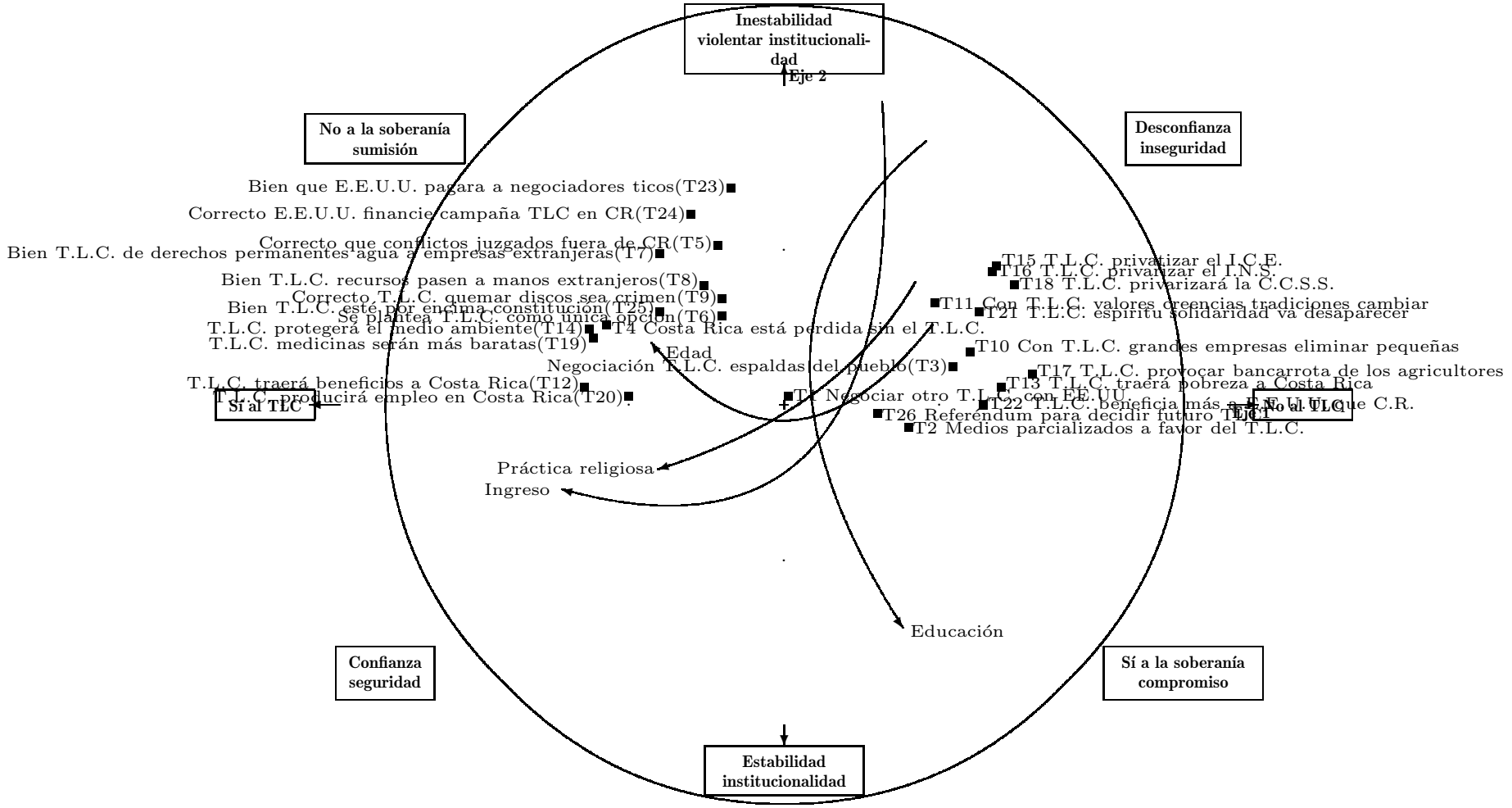




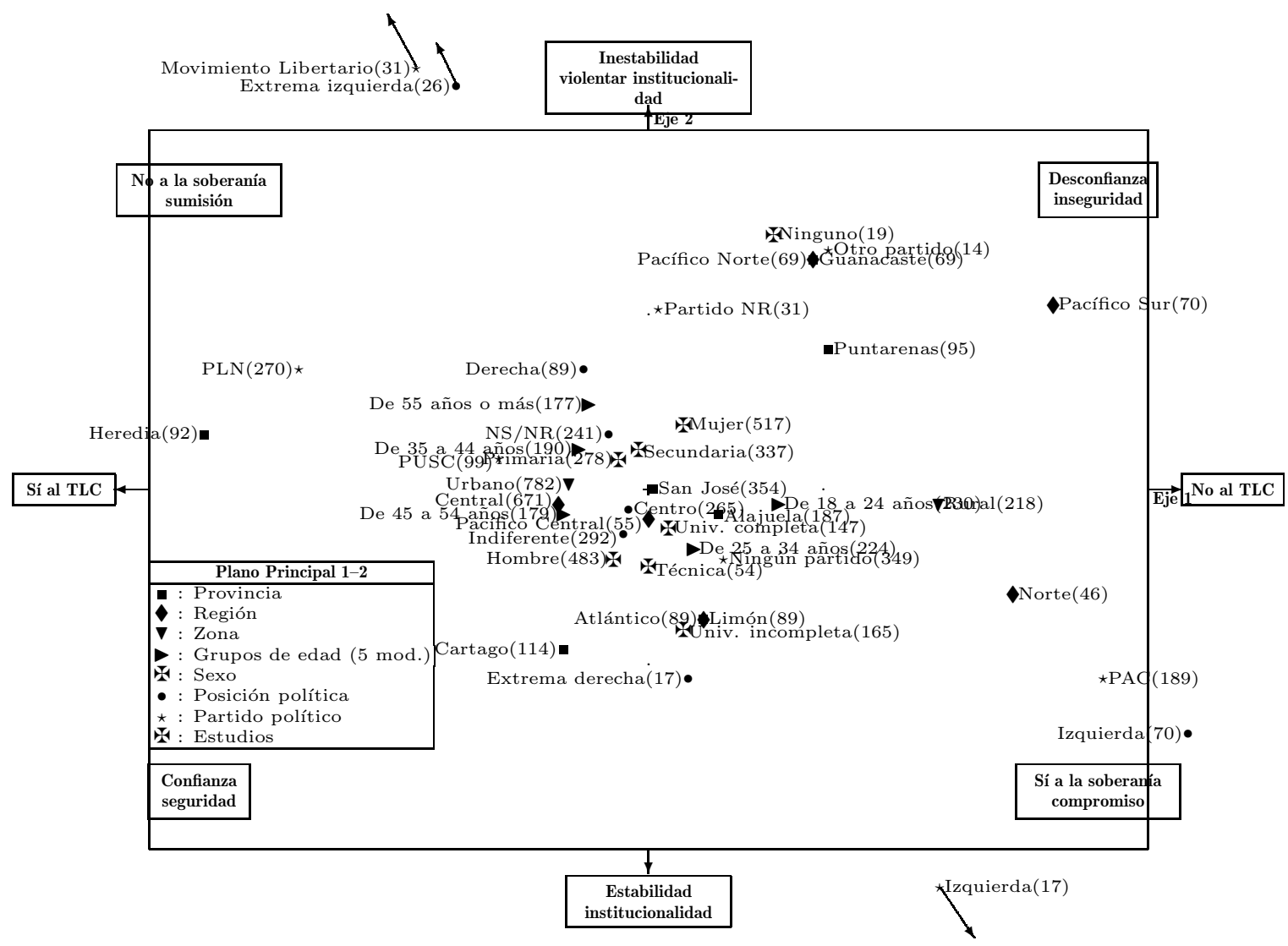

\begin{tabular}{l|l|r|r|r}
\hline \hline & & 2004 & 2005 & 2006 \\
\hline Está bien que TLC esté por encima de la Constitución & Contra & 66,2 & 70,1 & 69,2 \\
& Indif & 14,9 & 10,5 & 10,6 \\
& Favor & 14,4 & 12,9 & 13,2 \\
\multicolumn{1}{c|}{644} & NR & 4,5 & 6,5 & 7,0 \\
\hline Se debe hacer un referéndum para decidir el futuro del & Contra & & 11,0 & 12,1 \\
T.L.C. con EE.UU. & Indif & 14,6 & 14,2 \\
& Favor & & 69,8 & 67,4 \\
& NR & & 4,6 & 6,3 \\
\hline \hline
\end{tabular}


En cuanto a las consecuencias y situación del T.L.C. con E.E.U.U, por favor responda SI o NO en cada una de las siguientes afirmaciones:

\begin{tabular}{|c|c|c|c|c|c|c|c|c|c|}
\hline & \multicolumn{3}{|c|}{2004} & \multicolumn{3}{|c|}{2005} & \multicolumn{3}{|c|}{2006} \\
\hline & $\mathrm{Si}$ & No & $\mathrm{NS} / \mathrm{NR}$ & $\mathrm{Si}$ & No & $\mathrm{NS} / \mathrm{NR}$ & $\mathrm{Si}$ & No & $\mathrm{NS} / \mathrm{NR}$ \\
\hline Debe negociarse otro T.L.C. con EE.UU. & & & & & & & 54,8 & 34,8 & 10,4 \\
\hline $\begin{array}{l}\text { Los medios masivos de comunicación están } \\
\text { parcializados en favor del T.L.C. }\end{array}$ & & & & & & & 65,4 & 21,5 & 13,1 \\
\hline $\begin{array}{l}\text { La negociación del T.L.C. la hicieran a espal- } \\
\text { das del pueblo costarricense }\end{array}$ & & & & & & & 63,2 & 26,4 & 10,4 \\
\hline Costa Rica está perdida sin el T.L.C. & & & & & & & 25,6 & 62,6 & 11,8 \\
\hline $\begin{array}{l}\text { Es correcto que con el T.L.C. los conflictos en- } \\
\text { tre empresas extranjeras y el gobierno costar- } \\
\text { ricense, sean juzgados fuera de Costa Rica }\end{array}$ & & & & & & & 18,5 & 67,2 & 14,3 \\
\hline Se plantea al T.L.C. como la única opción & & & & & & & 47,9 & 43,1 & 9,0 \\
\hline $\begin{array}{l}\text { Está bien que el T.L.C. brinde derechos per- } \\
\text { manentes sobre las aguas que utilizan actual- } \\
\text { mente empresas extranjeras }\end{array}$ & & & & & & & 13,2 & 71,9 & 14,9 \\
\hline $\begin{array}{l}\text { Está bien que con el T.L.C. algunos recur- } \\
\text { sos naturales como las playas que actualmente } \\
\text { son de los costarricenses, pasen a manos ex- } \\
\text { tranjeras }\end{array}$ & & & & & & & 5,9 & 86,1 & 8,0 \\
\hline $\begin{array}{l}\text { Es correcto que con el T.L.C. las personas que } \\
\text { "Queman" discos pasan a ser criminales }\end{array}$ & & & & & & & 27,4 & 60,3 & 12,3 \\
\hline $\begin{array}{l}\text { Con el T.L.C. las grandes empresas elimi- } \\
\text { narán a las pequeñas }\end{array}$ & & & & & & & 67,3 & 22,9 & 9,8 \\
\hline $\begin{array}{l}\text { Con el T.L.C. nuestros valores, creencias y } \\
\text { tradiciones cambiarán }\end{array}$ & & & & & & & 46,0 & 43,7 & 10,3 \\
\hline El T.L.C. traerá beneficios a Costa Rica & 59,0 & 29,9 & 11,1 & 47,0 & 34,6 & 18,4 & 45,8 & 37,5 & 16,7 \\
\hline El T.L.C. traerá pobreza a Costa Rica & 41,3 & 45,9 & 12,8 & 45,0 & 39,6 & 15,4 & 45,9 & 37,5 & 16,6 \\
\hline El T.L.C. protegerá el medio ambiente & & & & & & & 20,4 & 60,6 & 19,0 \\
\hline El T.L.C. va a deteriorar el medio ambiente & 39,5 & 45,9 & 14,6 & 40,6 & 38,9 & 20,5 & & & \\
\hline El T.L.C. privatizará el ICE & 55,5 & 30,7 & 13,8 & 57,2 & 26,3 & 16,5 & 65,3 & 22,2 & 12,5 \\
\hline El T.L.C. privatizará el INS & 53,9 & 31,6 & 14,5 & 56,6 & 25,3 & 18,1 & 65,9 & 20,8 & $\overline{13,3}$ \\
\hline $\begin{array}{l}\text { El T.L.C. provocará la bancarrota de los } \\
\text { agricultores }\end{array}$ & 55,4 & 35,2 & 9,4 & 54,4 & 30,8 & 14,8 & 60,1 & 27,9 & 12,0 \\
\hline El T.L.C. privatizará la C.C.S.S. & 46,7 & 38,6 & 14,7 & 53,0 & 30,5 & 16,5 & 59,4 & 27,5 & 13,1 \\
\hline El T.L.C. encarecerá las medicinas & 52,0 & 36,8 & 11,2 & 47,6 & 36,6 & 15,8 & & & \\
\hline El T.L.C. las medicinas serán más baratas & & & & & & & 24,9 & 60,4 & 14,7 \\
\hline T.L.C. producirá desempleo en Costa Rica & & & & 39,1 & 47,3 & 13,6 & & & \\
\hline El T.L.C. producirá empleo en Costa Rica & & & & & & & 56,7 & 29,9 & 13,4 \\
\hline $\begin{array}{l}\text { Al llegar nuevas grandes empresas extranjeras } \\
\text { con el T.L.C., el espíritu de solidaridad irá } \\
\text { desapareciendo }\end{array}$ & & & & & & & 50,4 & 36,1 & 13,5 \\
\hline $\begin{array}{l}\text { El T.L.C. es un tratado que beneficia más a } \\
\text { E.E.U.U. que a Costa Rica }\end{array}$ & & & & 69,6 & 16,5 & 13,9 & 72,9 & 17,0 & 10,1 \\
\hline $\begin{array}{l}\text { Estuvo bien que los E.E.U.U. pagara sobre- } \\
\text { sueldos a negociadores costarricenses del } \\
\text { T.L.C. }\end{array}$ & & & & 11,3 & 72,5 & 16,2 & 9,8 & 77,7 & 12,5 \\
\hline $\begin{array}{l}\text { Es correcto que E.E.U.U. financie la campaña } \\
\text { en los medios a favor del T.L.C. en Costa Rica }\end{array}$ & & & & 18,3 & 67,1 & 14,6 & 14,3 & 73,2 & 12,5 \\
\hline
\end{tabular}




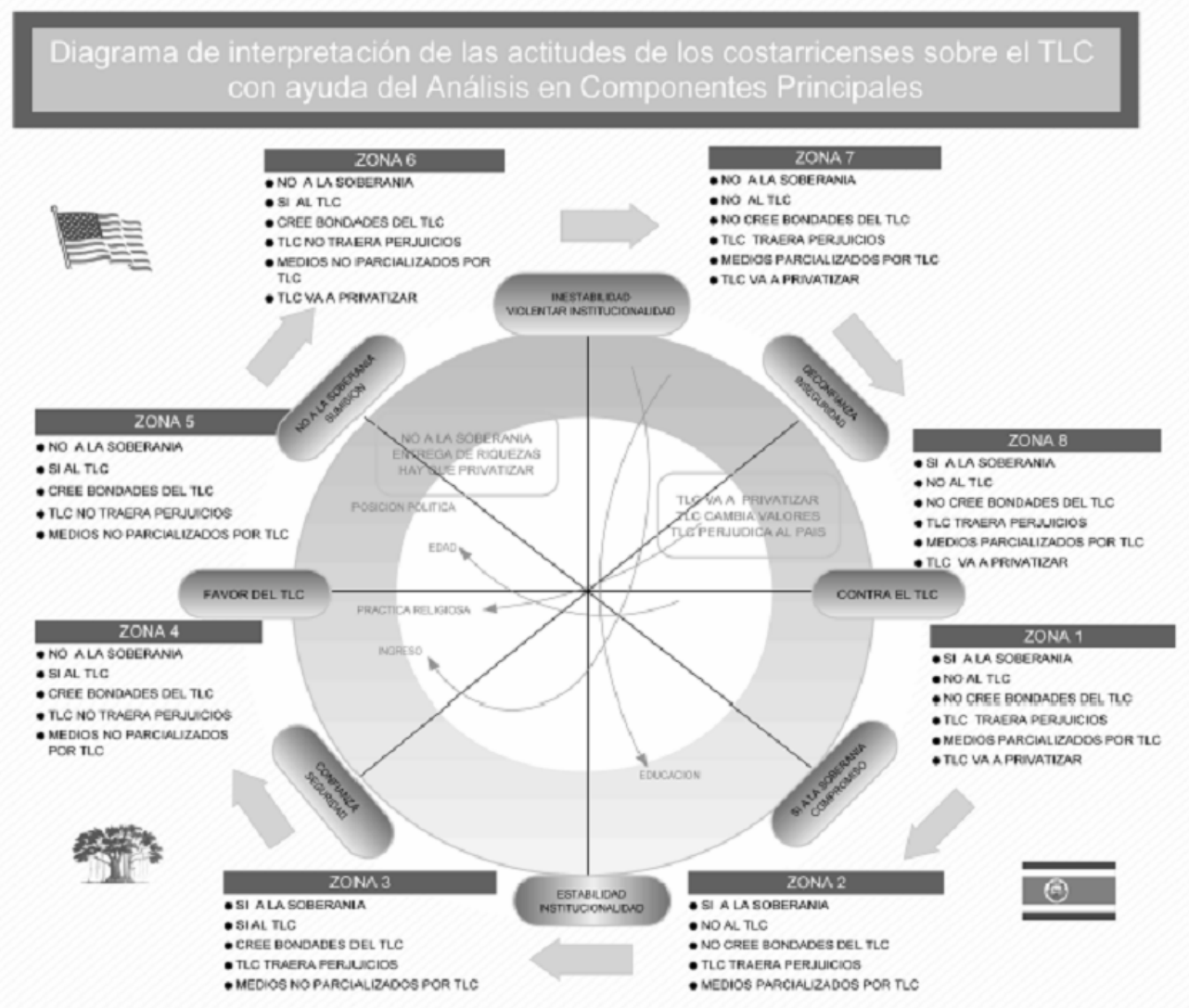

\section{Una reflexión sobre el TLC}

Como lo señala el Dr. Fernán Ulate de la Escuela de Economía en su intervención sobre los resultados de le encuesta, el T.L.C. no es simplemente un tratado de libre comercio, sino que es también una serie de lineamientos en política comercial, socio-económica y legal. Algunos de estos lineamientos abren un espectro de indefinición en aspectos esenciales para el futuro de los costarricenses, tales como la propiedad privada, los derechos de inversionistas extranjeros, la regulación efectiva del poder monopólico, etc. Para entender mejor estos conceptos analicemos algunas preguntas hechas en esta encuesta. La regulación legal de la zona marítimo terrestre se hace originalmente con una ley que tiene un espíritu nacionalista, pues pretende asegurar el acceso de los costarricenses a las playas, y al mismo tiempo brinda una serie de regulaciones que permiten a los costarricenses disfrutar de una propiedad frente al mar por un período específico, no siendo el dueño, pero teniendo derecho al uso y gozo de la misma hasta por 20 años. Sin embargo, contrariamente al espíritu de esta ley, no han sido los costarricenses sino los extranjeros los que más han disfrutado de este derecho. Se han construido propiedades que dentro de un tiempo, supuestamente, deberían pasar a los Gobiernos locales para que, de alguna manera, sean ellos los que las adjudiquen con su mejor criterio. Aquí hay un problema, pues en la mayoría de los casos, en una negociación con desigualdad de poder político-económico, 
los gobiernos municipales tendrán poca capacidad para negociar frente a los grandes millonarios e inversionistas extranjeros que actualmente están disfrutando de este derecho, de tal manera que el panorama abre una situación gris que terminará siendo definida por el poder político-económico, la capacidad de presionar, y las necesidades futuras de cada parte involucrada. Algo parecido sucederá con los recursos naturales. Veamos el caso específico del agua. La legislación costarricense dice claramente que es el Estado el que regula, adjudica y da concesiones para su uso. Estas concesiones necesitan una serie de estudios técnico-ambientales, que si bien es cierto son necesarios e imprescindibles para el buen manejo de los recursos naturales, deben ser pagados por el interesado y su costo es elevado. De tal manera que son los que tienen capacidad económica para pagarlos los que terminan con una serie de concesiones legales. Para el agricultor, comerciante o empresario costarricense de escasos recursos, pagar este tipo de estudios es prácticamente imposible. Después de una cierta cantidad de años en la que supuestamente la concesión vence, hay una serie de derechos adquiridos por el antiguo concesionario, que no están claramente regulados y definidos en nuestra legislación. La jurisprudencia que se está haciendo y la legislación que se haga en este sentido va a ser esencial para definir en el futuro los derechos de la propiedad e inversión extranjera sobre nuestros recursos naturales, y para garantizar a los costarricenses de todo nivel socio-económico igualdad en el acceso a estos recursos. Por otra parte, la política de subsidios a la agricultura por parte de Estados Unidos, complica mucho el entendimiento de la situación económica que se avecina. Por ejemplo, dados esos subsidios en alimentos como cereales, y a través del libre comercio, y dada la incapacidad de competir de los agricultores costarricenses con esos productos agrícolas subvencionados que vienen de países extranjeros, la experiencia podría ser apocalíptica. En este momento, podemos traer de afuera cereales y alimentos básicos para nuestra dieta, y a cambio exportar productos manufacturados porque las circunstancias de precios así lo permiten. Pero en el futuro, si estos alimentos van a ser usados para producir combustibles por ejemplo, el precio de ellos puede subir tanto que realmente condene a la hambruna a países enteros que desmantelaron su infraestructura productiva agrícola. El problema serio que la mayoría de los economistas teóricos no pueden entender, es que existe toda una cultura alrededor de la producción agrícola, que con excelente razón ha recibido el nombre de agricultura, y que esa cultura una vez destruida no puede recuperarse con la misma facilidad con la que se monta una enorme bodega para maquilar ropa (y que se traslada de un país a otro rápidamente de acuerdo a su conveniencia económica). En el trabajo "Formalización de una teoría de la mentalidad" (Fernán Ulate, por publicarse), se explica cómo la cultura es producto de un proceso evolutivo, y por qué se necesitan ciertas condiciones en la realidad histórica para que se dé el proceso específico que produjo esa cultura. Una vez desmantelada toda una actividad agrícola, se elimina, posiblemente para siempre, toda una cultura, una manera de ver el mundo, una serie de creencias, costumbres, espíritu de sacrificio, de trabajo bajo la lluvia, de trabajo bajo el sol ardiente, que se daba como producto de todo un proceso que se remonta a la colonia, y que fue gestado, adaptándose y aprovechando al máximo nuestra realidad geográfica, topográfica, calidad de suelos, clima y hasta tradiciones indígenas. Los países desarrollados comprenden muy bien esto, y por ello no van a permitir que se desmantele su agricultura. El tema de los monopolios es muy serio, entendiendo por monopolio no sólo el ICE,, el INS, 
la CCSS, sino todas aquellas empresas nacionales o extranjeras, que, gozando de un privilegio natural, económico, comercial, de derechos de autor y patentes, etc., restrinja la libre competencia de otras empresas que quieren entrar en ese tipo de mercado. ¿Están y tienen las entidades costarricenses encargadas de regular comportamientos monopólicos indeseables la capacidad real de ponerle límites a los abusos del monopolio, tal y como lo exige la Constitución Costarricense? Para poder ponerle límites a una corporación con excesivo poder económico, se necesita una legislación fuerte y sincera, así como entidades públicas con el poder, la capacidad y la voluntad, de cumplir con lo que exige la Constitución. Si esto no se hace adecuadamente y para rematar se hace un tratado en el cual sí se defienden los derechos de la inversión extranjera y multinacionales, entonces, unos años después el acceso a medicinas genéricas, de productos novedosos y necesarios para la salud, el acceso a materiales y software de computación, el acceso a toda la gama de descubrimientos biotecnológicos (incluyendo nuevos combustibles sustitutos del petróleo), el acceso a nano-tecnología, incluyendo nuevos materiales de construcción, tan importantes en un país afectado por sismos y terremotos como lo es Costa Rica, será limitado, y el equilibrio que exige la Constitución será cambiado por un desequilibrio a favor de las multinacionales y monopolios que sí tienen la capacidad económica, los asesores, y el poder político para sacarle provecho a nuestra falta de legislación efectiva y moderna, adaptada a las nuevas tecnologías. La encuesta demuestra la preocupación y desconfianza que tiene el pueblo costarricense en estos temas. El asunto de las medicinas genéricas, las semillas que producen plantas que a su vez producen semillas sin poder germinativo, el desinterés de nuestros países por las actitudes monopolistas de empresas que están siendo demandadas en Europa y en el mismo Estados Unidos, pero que quieren exigirle a los costarricenses que se les respete sus patentes y derechos de autor, la falta de conocimiento e ignorancia respecto a cómo las nuevas tecnologías afectarán nuestra economía y bienestar, son aspectos que representan la punta de un iceberg de un problema inconmensurable y hasta irreversible si no se hacen los cambios y se toman las medidas legislativas apropiadas.

\section{Referencias}

[1] Althusser, L. (1983) "Ideología y aparatos ideológicos del Estado", en La Filosofía como Arma de la Revolución. Cuadernos Pasado y Presente, No. 4, México.

[2] Anderson, T. W. (1958) Introduction to Multivariate Statistical Analysis. John Wiley \& Sons, New York.

[3] Anastassakos, I. (1984) Contributions à l'Histoire et a la Théorie du Modèle Factoriel. Thèse, Université Pierre et Marie Curie, Paris.

[4] Bartles, R. (1987) El placer del Texto y Lección Inaugural de la Cátedra de Semiología Literaria del College de France, 4a. edición ampliada. Editorial Siglo XXI, México.

[5] Cailliez, F.; Pagés, J.P. (1976) Introduction à l'Analyse des Données. Editorial SMASH, Paris.

[6] Gramsci, A. (1981) Cuadernos de la Cárcel 1. Edición Crítica del Instituto Gramsci, Ediciones ERA, México.

[7] Hinkelammert, F. (1981) Las Armas Ideológicas de la Muerte, 2a. edición. Editorial DEI, San Pedro.

[8] Horkheimer, M. (1974) Teoría Crítica. Amorrortu editores, Buenos Aires.

[9] Pagés, J.P. y colaboradores (1987) Les Structures de l'Opinión en 198\%. Ed. Agorametrie, Paris. 
[10] Pérez, M.; González, Y. (1987) "Reflexiones en torno a la comunicación actual: del discurso crítico a la resistencia cultural", inédito.

[11] Poltronieri, J.; Piza, E. (1989) Estructuras de Opinión Pública en Costa Rica. Ed. U.C.R., San José.

[12] Poltronieri, J. (1989) "La importancia del análisis en componentes principales en el estudio de la opinión pública", Informe presentado a la Caja Costarricense de Seguridad Social Feb. 1989, San José.

[13] Poltronieri, J. (1989) "Opinión pública y seguridad social", Informe presentado a la Caja Costarricense de Seguro Social, Nov. 1989, San José.

[14] González, J.; Poltronieri, J. (1990) "Estructuras de la opinión pública y la seguridad social”, Informe presentado a la Caja Costarricense de Seguro Social, San José.

[15] Poltronieri, J. (1991) "Contribución al estudio de la opinión pública", Ciencias Matemáticas 2(2).

[16] Poltronieri, J. (1992-1994) "Estructuras de la Opinión Pública en (1992-1994). Principales resultados estadísticos de la encuesta de (1992-1994)", informes presentados a la Vicerrectoría de Investigación de la Universidad de Costa Rica, San José.

[17] Garita, N.; Poltronieri, J. (1992) "Régimen de partidos políticos y opinión pública", Nov. 1992, San José.

[18] Garita, N.; Poltronieri, J. (1993) "Estructuras de la Opinión Pública y la Seguridad Social", informe presentado a la Caja Costarricense de Seguro Social, San José.

[19] Garita, N.; Poltronieri, J. (1995) Estructuras de la Opinión Pública en Costa Rica: ¿Estabilidad o evolución? Editorial Universidad de Costa Rica, San José.

[20] Garita, N. (2001) "La opinión pública como un espacio de diálogo", in: Estado de la Opinión Pública Costarricense: Un Análisis de los Principales Temas de Conflicto. Editorial CIMPA, San José, pag. 47-80.

[21] Poltronieri, J. (1995-2006) "Estructuras de la Opinión Pública en (1995-2006). Principales resultados estadísticos de la encuestas de (junio de 1989-junio 2006)", informe presentado a la Vicerrectoría de Investigación, Universidad de Costa Rica, San José.

[22] Poltronieri, J. (2000) "Estructuras de la Opinión Pública en 2000. Principales resultados estadísticos de la encuesta de junio de 2000", Informe presentado a la Caja Costarricense de Seguro Social, San José, 136 páginas.

[23] Poltronieri, J. (2001) "Fundamento Teórico-Metodológico del Estudio de la Opinión Pública", in: Estado de la Opinión Pública Costarricense: Un Análisis de los Principales Temas de Conflicto. Editorial CIMPA, San José, pag. 1-46.

[24] Poltronieri, J. (2006) "Encuesta de Opinión Pública 2006 - Panorama global", Proyecto de Investigación Estructuras de la Opinión Pública, Comunicado de prensa, setiembre 2006, pag. 1-74.

[25] Poltronieri, J. (2006) "Encuesta sobre corrupción en Costa Rica 2006", Proyecto de Investigación Estructuras de la Opinión Pública, Comunicado de prensa, setiembre 2006, pag. 1-59.

[26] Poulantzas, N. (1983) Poder Político y Clases Sociales en el Estado Capitalista, 20a. edición. S.XXI, México.

[27] Soto, W. (1987) Ideología y Medios de Comunicación Social en Costa Rica. Ed. Alma Mater, San José (Costa Rica).

[28] Lévi-Strauss, C. (1964) Mythologiques. Le Cru et le Cuit. Plon, Paris.

[29] Zeledón, M. (1987) La desinformación de la prensa en Costa Rica: un grave peligro para la paz. ICES, San José. 